\title{
Depression is highly prevalent but under-reported in children with ADHD
}

\author{
By Dr Jessica K Edwards
}

Researchers at Cardiff University have investigated whether the symptoms of depression observed in patients with attention-deficit/hyperactivity disorder (ADHD) differ from those reported in the general population. The study recruited 249 children with ADHD (mean age 14.6 years) and a large general population sample of 1460 individuals (mean age 12.8 years) to compare (i) the prevalence of depression symptoms, (ii) the depression symptoms most commonly reported and (iii) the child-reports and parent-reports of depression symptoms. Parents and children completed the Mood and Feelings Questionnaire (MFQ), which consists of descriptive phrases regarding how a subject has been feeling recently, and must be scored as either "true", "sometimes true" or "not true" 1. The total parent-reported MFQ and child-reported MFQ scores were then calculated and compared. The average parent-reported and child-reported MFQ scores were significantly higher in the ADHD sample compared to the general population, indicating that depression symptoms were more common in those with ADHD. The overall profile of depression symptoms in ADHD, however, was similar to the general population with feelings of irritability, restlessness, difficulties with concentration and being unhappy reported most often by both groups. Interestingly, parents of children with ADHD were more likely to report symptoms of depression than their child, whereas the reverse was true in the general population. This finding suggests that children with ADHD tend to under-report depression symptoms compared to the general population. The researchers conclude that depression symptoms are highly prevalent in ADHD, and exhibit a similar profile to the depression symptoms observed in the general population.

Fraser, A., Cooper, M., Agha, S. S., Collishaw, S., Rice, F., Thapar, A. \& Eyre,

$O$. (2017), The presentation of depression symptoms in attention-deficit/hyperactivity disorder: comparing child and parent reports. Child Adolesc Ment Health. doi:10.1111/camh.12253

\section{Further reading:}

${ }^{1}$ Angold, A. et al. (1995) The development of a short questionnaire for use in epidemiological studies of depression in children and adolescents. International Journal of Methods in Psychiatric Research, 5: 237 - 249.

\section{Glossary:}

Mood and Feelings Questionnaire (MFQ): A questionnaire containing 33 questions to capture the symptoms included in the DSM-IV criteria for major depressive disorders. It is considered an appropriate assessment tool for depression symptoms in children aged range 8-18 years, but not a method

to diagnose depression. The questions assess mood and anhedonia, tiredness, restlessness, concentration difficulties, and several aspects of negative self-evaluation. A high MFQ suggests more severe depressive symptoms. 